\title{
One-Way Speed of Light Relative to a Moving Observer
}

\author{
Stephan J. G. Gift ${ }^{1}$ \\ ${ }^{1}$ Department of Electrical and Computer Engineering, Faculty of Engineering, The University of the West Indies, \\ St. Augustine, Trinidad and Tobago, West Indies \\ Correspondence: Stephan J. G. Gift, Department of Electrical and Computer Engineering, Faculty of Engineering, \\ The University of the West Indies, St. Augustine, Trinidad and Tobago, West Indies. E-mail: \\ Stephan.Gift@sta.uwi.edu
}

\author{
Received: August 21, 2012 Accepted: September 10, 2012 Online Published: January 28, 2013 \\ doi:10.5539/apr.v5n1p135 \\ URL: http://dx.doi.org/10.5539/apr.v5n1p135
}

\begin{abstract}
The one-way speed of light relative to a moving observer is determined using the range equation of the Global Positioning System. This equation has been rigorously tested and verified in the Earth-Centred Inertial frame where light signals propagate in straight lines at constant speed $c$. The result is a simple demonstration of light speed anisotropy that is consistent with light speed anisotropy detected in other experiments and inconsistent with the principle of light speed constancy. This light speed anisotropy was not observed before because there has been no direct one-way measurement of light speed relative to a moving observer.
\end{abstract}

Keywords: special theory of relativity, principle of light speed constancy, GPS, range equation, one-way speed of light, moving observer, ECI frame

\section{Introduction}

The Global Positioning System (GPS) is a modern measurement system with accurate synchronized atomic clocks that enable precise navigation on the Earth $(\mathrm{Xu}, 2007)$. It has been hailed as a working example of the application of special relativity (Will, 2006). According to the IS-ICD-200 GPS Interface Specification (IS-GPS-200E, 2010), GPS signals propagate in straight lines at the constant speed $c$ in an Earth-Centered Inertial (ECI) frame, a frame that moves with the Earth but does not share its rotation. Wolf and Petit (1997) tested the isotropy of the speed of light in the ECI frame and published a limit of $\delta c / c<5 \times 10^{-9}$. This light speed isotropy in the ECI frame is utilized in the GPS range equation given by (Xu, 2007)

$$
\left|\bar{r}_{r}\left(t_{r}\right)-\bar{r}_{s}\left(t_{s}\right)\right|=c\left(t_{r}-t_{s}\right)
$$

where $t_{s}$ is the time of transmission of an electromagnetic signal from a source, $t_{r}$ is the time of reception of the electromagnetic signal by a receiver both times determined using synchronized clocks, $\bar{r}_{s}\left(t_{s}\right)$ is the position of the source at the time of transmission of the signal and $\overline{r_{r}}\left(t_{r}\right)$ is the position of the receiver at the time of reception of the signal. In this application, the source and/or the receiver can be in a state of motion relative to the ECI frame. The GPS clock synchronization procedure used in this system has been exhaustively tested and verified and results in clocks having the same time measurement for the same event.

Using elapsed time measurements determined by these synchronized GPS clocks and the light speed value $c$, Equation (1) allows accurate determination of the instantaneous position of objects which are stationary or moving on the surface of the Earth. It has been extensively and rigorously tested and verified and the system's very successful operation has resulted in the world-wide proliferation of GPS technology.

Light speed isotropy in the ECI frame and the appearance of $c$ in the range equation are sometimes interpreted as a demonstration of the principle of light speed constancy but this is not necessarily the case. This principle from Einstein's special theory of relativity holds that light speed is constant in all inertial frames (French, 1968; Rindler, 1991; Williams, 2002). It should be noted that even though the principle is stated as applying in inertial frames, the vast majority of light speed experiments claiming confirmation of the principle and its several important applications such as clock synchronization and the SI standard of length measurement involve the non-inertial frame of the rotating Earth (Zhang, 1997; Muller, 2003; Wolf, 2004; Hermann, 2005; Antonini, 2005; Eisele, 2009; Herrmann, 2009). Zhang (1997) has pointed out that two-way light speed isotropy in this frame has 
been confirmed while one-way light speed isotropy has not been legitimately examined in the few experiments (Gagnon, 1988; Krisher, 1990; Riis, 1988) in which such a test has been attempted. Spavieri (2012) recently proposed a new method for one-way light speed determination but this has not been tested.

In this regard, light speed anisotropy induced by the Earth's rotational motion has been directly demonstrated for East-West light travel using the synchronized clocks of the GPS (Marmet, 2000; Kelly, 2005; Gift, 2010a). Light speed variation arising from the Earth's orbital motion was also detected in the Roemer experiment involving occultations of Jupiter's satellite Io observed from the moving Earth (Gift, 2010b) and by Shtyrkov in the tracking of geostationary satellites (Shtyrkov, 2005). In this paper we supplement these findings by demonstrating light speed anisotropy in the ECI frame for a relatively moving observer utilizing the range equation of the GPS. Previously Gift (2011) used the range equation to demonstrate light speed variation for light travelling East-West on the surface of the Earth. Here we use the range equation to directly evaluate the one-way speed of light relative to an observer moving uniformly in the ECI frame, the light emanating from a source fixed in that frame.

\section{Light Speed Relative to a Moving Observer in the ECI Frame}

Consider a GPS station A fixed in the ECI frame and a GPS station B moving at a constant speed $v$ relative to the ECI frame. On an axis fixed in the ECI frame along the line joining the two stations with station A closer to the origin $\mathrm{O}$ than station $\mathrm{B}$ and taking positive values, let $x_{A}$ be the fixed position of station $\mathrm{A}$ and $x_{B}(t)$ be the position of station B at time $t$. At time $t_{I}$ let the distance between the two stations be $L$ given by

$$
x_{B}\left(t_{I}\right)-x_{A}=L
$$

\subsection{Observer Moving away from Fixed Source}

Let station B move directly away from fixed station A at a speed $v$ relative to the ECI frame. At time $t_{I}$ let station A transmit a signal directly to station $\mathrm{B}$ which receives it at time $t_{F}$. Then from the range equation (1),

$$
x_{B}\left(t_{F}\right)-x_{A}=c\left(t_{F}-t_{I}\right)
$$

where $x_{B}\left(t_{F}\right)$ is the position of station B at time $t_{F}$. Since station B is moving uniformly away from station A at speed $v$ relative to the ECI frame, it follows that the relation between the position $x_{B}\left(t_{F}\right)$ of station $\mathrm{B}$ at the time of reception of the signal and its position $x_{B}\left(t_{I}\right)$ at the time of emission of the signal is given by

$$
x_{B}\left(t_{F}\right)=x_{B}\left(t_{I}\right)+v\left(t_{F}-t_{I}\right)
$$

Substituting for $x_{B}\left(t_{F}\right)$ from (4) in (3) yields

$$
x_{B}\left(t_{I}\right)-x_{A}+v\left(t_{F}-t_{I}\right)=c\left(t_{F}-t_{I}\right)
$$

Using (2) this becomes

$$
x_{B}\left(t_{I}\right)-x_{A}=L=(c-v)\left(t_{F}-t_{I}\right)
$$

Hence for an observer on station $B$ the range equation gives the elapsed time as

$$
\left(t_{F}-t_{I}\right)=\frac{L}{c-v}
$$

Therefore the speed $c_{A B}$ of the light relative to station B for the light traveling from the fixed station A to the moving station B is given by initial separation $L$ divided by elapsed time $\left(t_{F}-t_{I}\right)$ which using (7) is

$$
c_{A B}=\frac{L}{\left(t_{F}-t_{I}\right)}=\frac{L}{L /(c-v)}=c-v
$$

This light speed $c_{A B}=c-v$ observed at station $\mathrm{B}$ is unaffected by "relativistic" changes in the direct measurement of $L$ by the moving observer which are second-order. It is a clear indication of light speed variation arising from movement of the observer away from the source fixed in the ECI frame and a direct negation of the principle of light speed constancy which demands that the moving observer detect light speed $c_{A B}=c$.

\subsection{Observer Moving toward Fixed Source}

Let station B move directly toward fixed station A at a speed $v$ relative to the ECI frame. At time $t_{I}$ let station A transmit a signal directly to station $\mathrm{B}$ which receives it at time $t_{F}$. Then using the range equation (1) and noting that $x_{B}\left(t_{F}\right)>x_{A}$, 


$$
x_{B}\left(t_{F}\right)-x_{A}=c\left(t_{F}-t_{I}\right)
$$

Since station B is moving uniformly toward station A at speed $v$ relative to the ECI frame, it follows that the relation between the position $x_{B}\left(t_{F}\right)$ of station $\mathrm{B}$ at the time of reception of the signal and its position $x_{B}\left(t_{I}\right)$ at the time of emission of the signal is given by

$$
x_{B}\left(t_{F}\right)=x_{B}\left(t_{I}\right)-v\left(t_{F}-t_{I}\right)
$$

Substituting for $x_{B}\left(t_{F}\right)$ from (10) in (9) yields

$$
x_{B}\left(t_{I}\right)-x_{A}-v\left(t_{F}-t_{I}\right)=c\left(t_{F}-t_{I}\right)
$$

Using (2) this becomes

$$
x_{B}\left(t_{I}\right)-x_{A}=L=(c+v)\left(t_{F}-t_{I}\right)
$$

Hence for an observer on station $B$ the range equation yields the elapsed time as

$$
\left(t_{F}-t_{I}\right)=\frac{L}{c+v}
$$

Therefore the speed $c_{A B}$ of the light relative to station $\mathrm{B}$ for the light traveling from the fixed station A to the moving station B is given by initial separation $L$ divided by elapsed time $\left(t_{F}-t_{I}\right)$ which using (13) is

$$
c_{A B}=\frac{L}{\left(t_{F}-t_{I}\right)}=\frac{L}{L /(c+v)}=c+v
$$

Again, this light speed $c_{A B}=c+v$ observed at station B is unaffected by "relativistic" changes in the direct measurement of $L$ by the moving observer which are second-order. It is a clear indication of light speed variation arising from movement of the observer toward the source fixed in the ECI frame and a direct invalidation of the principle of light speed constancy which requires that the moving observer measure light speed $c_{A B}=c$.

\subsection{Discussion}

The elapsed times determined in Equations (7) and (13) indicate that the light transmitted from station A takes longer to arrive at station B when station B is moving away from A than towards it. The availability of synchronized clocks in the GPS enables the East-West times $\left(t_{F}-t_{I}\right)$ to be actually measured and the elapsed times given in (7) and (13) are exactly the times that would be measured in such a test using these synchronized GPS clocks. Following from this Equations (8) and (14) indicate that for an observer moving at a constant speed $v$ relative to the ECI frame, the speed of light from a source fixed in the ECI frame relative to that moving observer is $c-v$ for the observer moving away from the source and $c+v$ for the observer moving towards the source. Similar light speed changes $c \pm w$ for a moving observer were reported in the Roemer (Gift, 2010b) and Shtyrkov (2005) experiments involving the orbital movement of the Earth at speed $w$ in its approximately linear motion around the Sun.

The light speeds $c \pm v$ for a moving observer determined using the GPS range equation in the ECI frame were not detected by the many light speed experiments conducted in the terrestrial frame which all give light speed $c$. These changed light speed values $c \pm v$ observed in an inertial frame directly contradict the principle of light speed constancy which requires constant light speed $c$ that is independent of the uniform motion of the observer.

Additionally the operational accuracy of the range equation in the ECI frame and the detection of light speed anisotropy $c \pm v$ in inertial frames moving relative to the ECI frame indicate that light travels at speed $c$ in the ECI frame only and travels at different speeds in frames moving relative to the ECI frame. The ECI frame therefore behaves as a preferred frame for the propagation of light and it is ironic that the "incredible accuracy" of this "modern radio navigational system [GPS]" is cited by Rindler as an indication of the absence of a preferred frame (Rindler, 2006).

Why was this light speed anisotropy, so easily revealed by GPS technology, not observed before considering the numerous light speed experiments that have been conducted over the past century? The simple answer appears to be that there has previously been no direct one-way measurement of light speed with respect to a moving observer among the many that have been performed (Zhang, 1997; Gezari, 2009). The anisotropy result confirmed by this and previous work (Marmet, 2000; Kelly, 2005; Gift, 2010a) therefore represents an important development in space-time research that should be given serious attention by the scientific community. 
A related result presented by Gift (2011) using the range equation of the GPS in which the speed of light travelling East-West relative to the surface of the Earth is shown to be $c \pm v$ was recently challenged by Sharlanov (2011). However Sharlanov did not deduce light speed relative to the surface of the Earth but rather light speed in "the space where the light is propagated" which corresponds to the ECI frame in which the speed is $c$. His challenge is therefore invalid and the light speed result $c \pm v$ in Gift (2011) stands.

\section{Conclusion}

In this paper, elementary analysis involving the rigorously verified range equation of the GPS was used to determine the one-way speed of light in the ECI frame between a point fixed in the ECI frame and an observer moving at constant speed $v$ relative to the ECI frame.

The basis of this is that the GPS range equation applies to objects that are stationary or moving relative to the ECI and the GPS synchronized clocks enable the determination of time in the frames of those objects. Thus while in GPS navigation these measured times are used in the range equation to determine position, in this paper known positions $x_{A}, x_{B}\left(t_{I}\right)$ and $x_{B}\left(t_{F}\right)$ were used in the range equation to determine time in the form of elapsed time $\left(t_{F}-t_{I}\right)$ in (7) and (13). Because the range equation has been rigorously and exhaustively tested and verified [GPS works!], these elapsed times (7) and (13) are exactly the times that would be measured in an actual test using the GPS clocks. The speed calculation given in Equations (8) and (14) then follows from elementary kinematics.

The detected light speed anisotropy $c \pm v$ is a clear demonstration of light speed variation in the ECI frame arising from the movement of the observer and is consistent with anisotropy results previously obtained in the terrestrial frame (Marmet, 2000; Kelly, 2005; Gift, 2010a). It contradicts the principle of light speed constancy which is formulated in inertial frames and is today routinely applied in the non-inertial frame of the surface of the Earth. This particular source of light speed anisotropy was not observed before perhaps because there has been no direct one-way measurement of light speed with respect to a moving observer.

This significant result obtained using accurate GPS technology further supports the finding (Gift, 2009) that the Absolute Space Theory of the Selleri (Inertial) Transformations is the best description of space and time, and that Special Relativity Theory based on the Lorentz Transformations along with all theories that are derived from members of the complete set of "equivalent" space-time transformations given by Selleri $(2004,2011)$ are invalid representations of the physical world.

\section{References}

Antonini, P., Okhapkin, M., Goklu, E., \& Schiller, S. (2005). Test of Constancy of Speed of Light With Rotating Cryogenic Optical Resonators. Physical Review A, 71, 050101. http://dx.doi.org/10.1103/PhysRevA.71.050101

Eisele, C, Nevsky, A., \& Schiller, S. (2009). Laboratory Test of the Isotropy of Light Propagation at the 10-17 Level. Physical Review Letters, 103, 090401. http://dx.doi.org/10.1103/PhysRevLett.103.090401

French, A. P. (1968). Special Relativity. Nelson, London.

Gagnon, D. R., Torr, D. G., Kolen, P. T., \& Chang, T. (1988). Guided-Wave Measurement of the one-way Speed of Light. Physical Review A, 38, 1767. http://dx.doi.org/10.1103/PhysRevA.38.1767

Gezari, D. Y. (2009). Experimental Basis for Special Relativity in the Photon Sector. arXiv: 0912.3818.

Gift, S. J. G. (2009). Separating Equivalent Space-Time Theories. Apeiron, 16, 408.

Gift, S. J. G. (2010a). One-Way Light Speed Measurement Using the Synchronized Clocks of the Global Positioning System (GPS). Physics Essays, 23, 271. http://dx.doi.org/10.4006/1.3361840

Gift, S. J. G. (2010b). Light Speed Invariance is a Remarkable Illusion. Physics Essays, 23, 1. http://dx.doi.org/10.4006/1.3280803

Gift, S. J. G., (2011). One-Way Light Speed Determination Using the Range Measurement Equation of the GPS. Applied Physics Research, 3, 110. http://dx.doi.org/10.5539/apr.v3n1p110

Herrmann, S., Senger, A., Kovalchuk, E., Muller, H., \& Peters, A. (2005). Test of the Isotropy of the Speed of Light Using a Continuously Rotating Optical Resonator. Physical Review Letters, 95, 150401. http://dx.doi.org/10.1103/PhysRevLett.95.150401

Herrmann, S., Senger, A., Mohle, K., Nagel, M., Kovalchuk, E. V., \& Peters, A. (2009). Rotating optical cavity experiment testing Lorentz invariance at the 10-17 level. Physical Review D, 80, 105011. http://dx.doi.org/10.1103/PhysRevD.80.105011 
$\begin{array}{lllllll}\text { IS-GPS-200E. (2010). } & \text { P. } & \text { 95. Retrieved } & \text { May } & \text { 17, 2012, } & \text { from }\end{array}$ http://www.gps.gov/technical/icwg/IS-GPS-200E.pdf

Kelly, A. (2005). Challenging Modern Physics. Florida: BrownWalker Press.

Krisher, T. P., Maleki, L., Lutes, G. F., Primas, L. E., Logan, R. T., Anderson, J. D., \& Will, C. M. (1990). Test of the Isotropy of the One-way Speed of Light Using Hydrogen-Maser Frequency Standards. Physical Review $D, 42,731$. http://dx.doi.org/10.1103/PhysRevD.42.731

Marmet, P. (2000). The GPS and the Constant Velocity of Light. Acta Scientiarum, 22, 1269.

Muller, H., Herrmann, S., Braxmaier, C., \& Peters, A. (2003). Modern Michelson-Morley Experiment using Cryogenic Optical Resonators. Physical Review Letters, 91, 020401. http://dx.doi.org/10.1103/PhysRevLett.91.020401

Riis, E., Lars-Ulrik, A. A., Bjerre, N., \& Poulsen, O. (1988). Test of the Speed of Light Using Fast-Beam Laser Spectroscopy. Physical Review Letters, 60, 81. http://dx.doi.org/10.1103/PhysRevLett.60.81

Rindler, W. (1991). Introduction to Special Relativity. Oxford: Clarendon Press.

Rindler, W. (2006). Relativity Special, General and Cosmological (2nd ed.). Oxford: Oxford University Press.

Selleri, F. (2004). Recovering the Lorentz Ether. Apeiron, 11, 246.

Selleri, F. (2011). La Relativita Debole, Edizioni Melquiades, Milano.

Sharlanov, G. V. (2011). The Influence of Gravitation on the Speed of Light and an Explanation of the Pioneer 10 \& 11 Acceleration Anomaly. Applied Physics Research, 3(2), 241. http://dx.doi.org/10.5539/apr.v3n2p241

Shtyrkov, E. I. (2005). Observation of Ether Drift in Experiments with Geostationary Satellites. Proceedings of the Natural Philosophy Alliance, pp. 201-205, 12th Annual Conference, Storrs CT, 23-27 May, Space Time Analyses Ltd., MA.

Spavieri, G. (2012). On Measuring the One-way Speed of Light. The European Physics Journal D, 66, 76. http://dx.doi.org/10.1140/epjd/e2012-20524-8

Will, C. M. (2006). Special Relativity: A Centenary Perspective. Progress in Mathematical Physics, 47, 33.

Williams, W. S. C. (2002). Introducing Special Relativity. London: Taylor and Francis.

Wolf, P., \& Petit, G. (1997). Satellite Test of Special Relativity Using the Global Positioning System. Physical Review A, 56, 4405. http://dx.doi.org/10.1103/PhysRevA.56.4405

Wolf, P., Bize, S., Clairon, A., \& Santarelli, G. (2004). Improved Test of Lorentz Invariance in Electrodynamics. Physical Review D, 70, 051902. http://dx.doi.org/10.1103/PhysRevD.70.051902

Xu, G. (2007). GPS Theory, Algorithms and Applications (2nd ed.). Springer-Verlag, Berlin.

Zhang, Y. Z. (1997). Special Relativity and its Experimental Foundations. Singapore: World Scientific. 